BULl. AUSTRAL. MATH. SOC.

VOL. 33 (1986), 155-156.

\title{
SLENDER BODY THEORY NEAR AN INTERFACE AT VERY LOW REYNOLDS' NUMBER GLENN ROBERT FULFORD
}

This dissertation contributes to an improved understanding of the mechanics of a particle, moving in the vicinity of an interface between two viscous fluids, by considering several fundamental problems involving slender bodies. In these problems it is assumed that the Reynolds' number is very small. Several useful applications of this study are discussed, including a theoretical model of muco-ciliary transport in the lung, where the force acting on a cilium near an interface is of primary importance.

One problem considered is that of a slender body translating near a flat interface, where the centreline of the slender body is oriented either parallel or perpendicular to the interface. The force distributions and the total drag force acting on the body are evaluated. For motion normal to the interface, the drag increases as the body gets closer to the interface, whereas for parallel motion, the drag increases or decreases depending on the ratio of viscosities of the fluids. The external couple, required to prevent the body from rotating when it moves parallel to the interface, is calculated. It is observed that two different mechanisms are responsible for the interface induced rotation of a particle, corresponding to parallel and perpendicular orientations of the slender body. A first order approximation of the interface deformation is also evaluated.

Received 16 July 1985. Thesis submitted to University of Wollongong, December 1984. Degree approved April 1985. Supervisor: Prof. Blake.

Copyright Clearance Centre, Inc. Serial-fee code: 004-9727/86 $\$ A 2.00+0.00$. 
This study is extended by considering the force distribution acting on a slender body which is straddling the interface between two fluids. It is assumed that the axis of the slender body is always held pexpendicular to the undisturbed interface. A shielding region is observed about the interface. Outside the shielding region the interaction of the slender body in one fluid with that part of the slender body in the other fluid is small compared to the interaction with the interface. The first order interface deformation is calculated for motion of the slender body parallel to the interface.

A mathematical model describing mucous transport in the lung is developed where the cilia may penetrate into a very viscous mucous layer. The results are particularly sensitive to the form of the cilia beat pattern. They also predict that the mucous velocity is not significantly increased when the cilia penetrate into the mucous layer, provided the active cilia density is high.

Department of Mathematics

La Trobe University

Bundoora, Victoria 3083

Australia. 